\title{
Disfagia cervical espondilótica por hiperostosis esquelética difusa idiopática en un paciente joven
}

\author{
JULIO URRUTIA, ANDRÉS BERNARDÍN, \\ CRISTIÁN MORALES, RODRIGO MILLÁN
}

\section{Diffuse idiopathic skeletal hyperostosis causing dysphagia in a young patient}

\begin{abstract}
Diffuse idiopathic skeletal hyperostosis (DISH) is an under-diagnosed condition producing flowing ossification of the antero-lateral ligaments of the spine. Affecting predominantly males over 65 years old, it is an unusual cause of dysphagia and dysphonia. We report a 45-year-old male with a three years history of dysphonia and three months of dysphagia. The initial diagnosis was gastroesophageal reflux, and an endoscopy ruled out esophageal luminal pathology. Cervical spine radiographs showed ossification of the cervical anterior longitudinal ligament with large, prominent osteophytes from $\mathrm{C} 3$ to $\mathrm{C6}$, producing esophageal and upper airway compression; these images were compatible with DISH. Cervical osteophyte resection resulted in complete resolution of symptoms. DISH should be considered in the differential diagnosis of dysphagia and dysphonia.
\end{abstract}

(Rev Med Chile 2013; 141: 803-806).

Key words: Deglutition disorders; Hyperostosis, diffuse idiopathic skeletal.

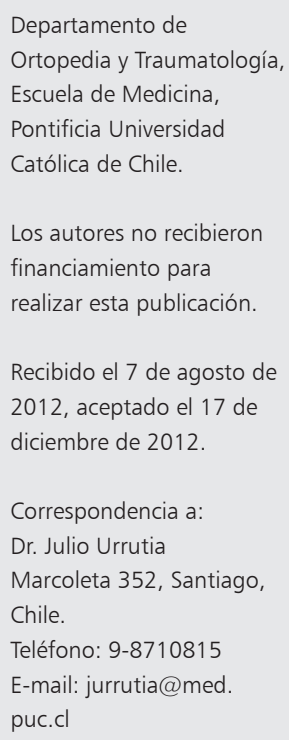

L a hiperostosis esquelética difusa idiopática, cuyo acrónimo en inglés es DISH (diffuse idiopatic skeletal hyperostosis), es una entesopatía no inflamatoria que lleva a la osificación de ligamentos y sitios de inserción en la columna vertebral y también del esqueleto apendicular. Los criterios para el diagnóstico son tres: calcificación/ osificación del aspecto ánterolateral de cuatro cuerpos vertebrales contiguos, preservación de la altura del disco intervertebral en el área comprometida, y ausencia de compromiso de las articulaciones interapofisiarias y sacroilíacas ${ }^{1-3}$. Esta condición, también conocida como enfermedad de Forestier, afecta preferentemente a hombres después de la séptima década de la vida, siendo el segmento torácico el más frecuentemente comprometido, seguido por el lumbar, siendo menos frecuente el compromiso cervical. Si bien la mayoría de los pacientes con compromiso cervical son asintomáticos o sólo presentan limitación de la movilidad o dolor inespecífico, existen distintos reportes de pacientes con síntomas secundarios a la compresión del esófago y la vía aérea como disfagia, estridor, disfonía y dificultades con la intubación o endoscopia ${ }^{2,4}$. Aunque una revisión sistemática reciente planteó que la disfagia y la disfonía en esta patología no serían tan raras como se había sugerido en reportes previos, dado que muchos médicos no están familiarizados con las características clínicas o radiológicas de este cuadro, el diagnóstico resulta difícil y muchas veces retrasado ${ }^{4}$.

Hasta la fecha, sólo tres casos de pacientes de hasta 45 años con este cuadro han sido reportados en la literatura internacional ${ }^{4}$; a continuación reportamos el caso de un paciente joven con disfonía y disfagia persistentes secundarios a DISH. 


\section{Caso}

Paciente de 45 años, sin antecedentes mórbidos, con historia de tres años de disfonía progresiva, asociada a disfagia en los últimos tres meses. Evaluado en múltiples ocasiones por otorrinolaringología, sin tener diagnóstico, fue manejado por fonoaudiología sin éxito; dado que se planteó el reflujo gastro-esofágico como causa de la disfonía, el paciente fue derivado a cirujano digestivo, quien en una endoscopia digestiva alta descartó la presencia de lesiones luminales, encontrando signos de compresión esofágica extrínseca, siendo manejado en forma sintomática sin éxito.

En relación a un episodio de cervicalgia sin irradiación, en que destacaba al examen una ausencia completa de flexión lateral de la columna cervical, el paciente fue estudiado con radiografías de columna cervical; éstas evidenciaron la presencia de una importante osificación del ligamento longitudinal anterior desde C3 a C6, con grandes osteofitos y preservación de la altura de los discos intervertebrales, con signos de compresión esofágica y de vía aérea alta (Figura 1). Habiéndose descartado compromiso sacroilíaco, se diagnosticó cuadro de DISH. El estudio se completó con un esofagograma que demostró una compresión extrínseca posterior de la hipofaringe y esófago superior por gran osteofito que comprometía los cuerpos vertebrales de C4 a C6 (Figura 2), y laringoscopia para descartar compromiso de cuerdas vocales por lesión del nervio laríngeo recurrente (LNR). Dada la compresión extrínseca del esófago y laringe secundarios a osteofitos cervicales por DISH, y considerando los síntomas de larga data, se decidió tratamiento quirúrgico.

A través de un abordaje anterior de SmithRobinson, y colocando una sonda naso-gástrica para identificar el esófago, se expuso el aspecto anterior de la columna cervical, realizándose una resección completa de los osteofitos anteriores y laterales con gubia de Leksell y fresa circular de alta velocidad, como fue descrito por Urrutia y col. ${ }^{5}$. El paciente evolucionó sin complicaciones, y la radiografía de control confirmó una remoción completa de osteofitos cervicales (Figura 3).

En control post operatorio, el paciente ha evolucionado asintomático, sin disfagia, y al mes presentaba regresión completa de los síntomas, lo que se ha mantenido a los seis meses.

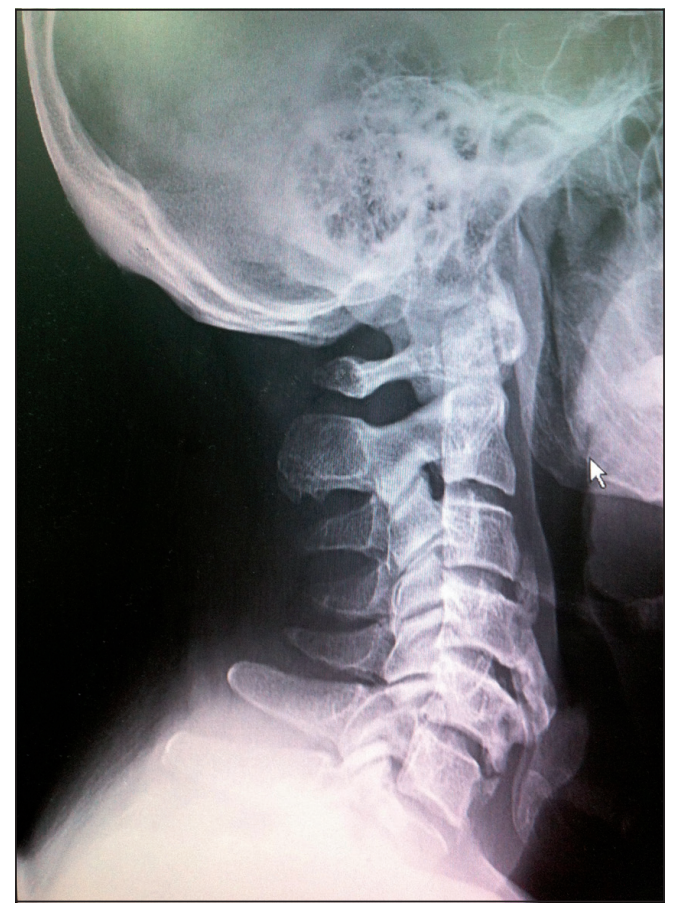

Figura 1. Radiografía lateral de columna cervical que evidencia grandes osteofitos de C3 a C6, con signos de compresión esofágica y de vía aérea alta.

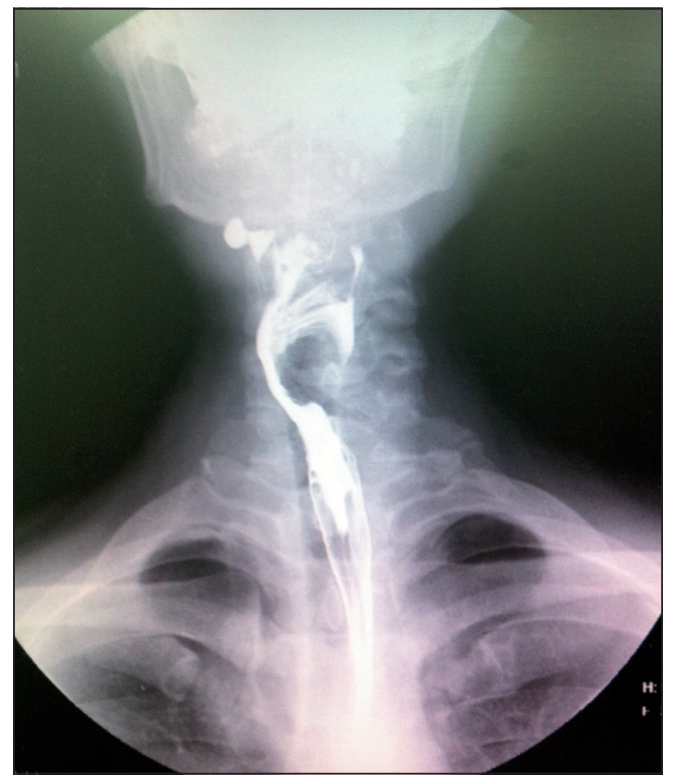

Figura 2. Esofagograma mostrando compresión extrínseca del esófago por gran osteofito que limita el paso del bario. 


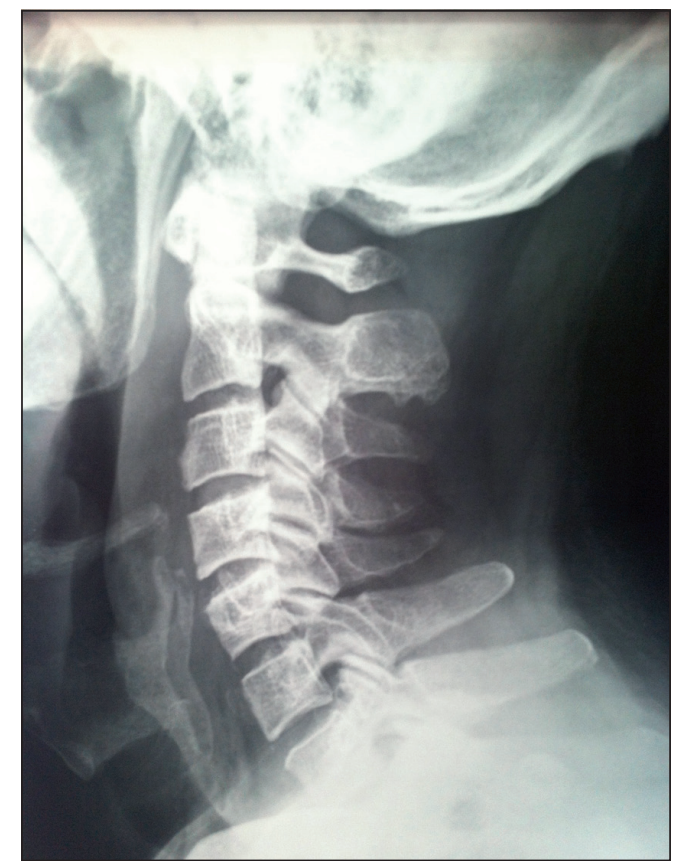

Figura 3. Radiografía postoperatoria que muestra remoción completa de osteofitos cervicales y de compresión esofágica y de vía aérea.

\section{Discusión}

Los reportes de disfagia o la obstrucción de la vía respiratoria alta como consecuencia de DISH han sido infrecuentes, si bien, esto puede representar una subestimación del número real de casos. En una revisión sistemática reciente ${ }^{4}$ se encontraron 118 artículos con un total de 204 pacientes reportados con este cuadro, correspondiendo la mayoría a publicaciones en el área de la cirugía de columna u otorrinolaringología, pese a que estos pacientes consultan primariamente a médicos generales, internistas o gastroenterólogos.

La mayoría de estos pacientes presentan disfagia alta, con sensación de dificultad al paso de alimentos sólidos y, muchas veces, tos al deglutir, además de síntomas de vía aérea alta como disfonía, estridor e incluso disnea. En la fisiopatología de la disfagia no sólo se plantea la compresión mecánica del esófago por osteofitos en una zona relativamente fija del esófago, sino también la presencia de edema de la pared esofágica por irritación en relación a las calcificaciones, alteración del peristaltismo y consecuentemente una dinámica de la deglución anormal ${ }^{2,6-8}$; incluso, la compresión mantenida del esófago puede llevar a daño del plexo neural esofágico ${ }^{9}$ y del NLR $^{10}$.

Aunque la edad promedio de presentación de los pacientes con disfagia u obstrucción de la vía respiratoria secundaria a DISH alcanza los 69 años, $11 \%$ de los pacientes son menores de 56 años ${ }^{4}$. Se ha postulado que el crecimiento lento de los osteofitos cervicales es bien tolerado por la mayoría de los individuos, aunque tendrían poca capacidad de compensación frente a eventos agudos como aspiración, regurgitación, asfixia, apnea del sueño, infección del tracto respiratorio superior, o trauma menor cervical ${ }^{11}$; estos eventos pueden producir aumento de volumen de los tejidos blandos alrededor del esófago y tráquea, llevando a la aparición de síntomas. Esto explicaría la lógica de intentar un tratamiento médico inicial en todos los casos.

La evaluación de estos pacientes debiera incluir a) una evaluación clínica completa de las estructuras orofaríngeas y rango de movilidad cervical; b) una radiografía lateral de columna cervical que permita demostrar la presencia de osificación entre los cuerpos vertebrales y su extensión, así como estimar el grosor de las partes blandas retrofaríngeas, y c) un esofagograma con ingesta oral de bario y un estudio en ántero-posterior y lateral que permita evaluar el paso del bario por el esófago y su eventual paso a la vía aérea alta²

La mayoría de los pacientes responde al tratamiento conservador, basado en la modificación del régimen y el uso de anti-inflamatorios no esteroidales, que busca reducir la inflamación asociada y mejorar la mecánica de la deglución ${ }^{2,8}$. La necesidad de tratamiento quirúrgico se reserva sólo para los casos que no responden a tratamiento médico, aunque se ha descrito ampliamente que la resección de los osteofitos permite aliviar la compresión mecánica y revertir los síntomas en la mayoría de los pacientes ${ }^{4,5,12,13}$, con un resultado que se mantiene a largo plazo $^{5}$.

La mayoría de los reportes sobre tratamiento quirúrgico en estos casos han usado un abordaje cervical anterior de Smith-Robinson para la resección de osteofitos, como el que describimos en este reporte ${ }^{14-16}$, aunque también se ha descrito el uso del abordaje transoral/transfaríngeo ${ }^{17}$, el que presenta un nivel de complejidad y riesgo muy superior.

$\mathrm{Al}$ ser una condición poco conocida, el DISH rara vez es planteado en el diagnóstico diferencial de los pacientes con disfagia o disfonía; de hecho, 
en un estudio reciente en los Países Bajos que mostró una prevalencia de $17 \%$ de DISH en 501 radiografías de tórax de pacientes ambulatorios, el diagnóstico estuvo ausente en todos los informes radiológicos originales ${ }^{18}$; esto sugiere el desconocimiento de las características radiológicas de esta patología, incluso entre radiólogos en un entorno académico, por lo que es importante aumentar la sospecha diagnóstica.

El interés del caso que reportamos consiste en la aparición de síntomas crónicos en un paciente joven, lo que sólo ha sido reportado sólo en tres ocasiones en la literatura internacional ${ }^{4}$, explicando la dificultad y demora en el diagnóstico en este caso en particular.

El DISH como causa de disfagia u obstrucción de la vía aérea alta puede ser un fenómeno sub-diagnosticado, por lo que sugerimos que debe considerarse en el diagnóstico diferencial de pacientes mayores de 65 años con estos síntomas, pero incluso en pacientes jóvenes como el que reportamos.

\section{Referencias}

1. Mizuno J, Nakagawa H, Song J. Symptomatic ossification of the anterior longitudinal ligament with stenosis of the cervical spine: a report of seven cases. J Bone Joint Surg Br 2005; 87: 1375-9.

2. Masiero S, Padoan E, Bazzi M, et al. Dysphagia due to diffuse idiopathic skeletal hyperostosis: an analysis of five cases. Rheumatol Int 2010; 30: 681-5.

3. Resnick D. Diffuse idiopathic skeletal hyperostosis. AJR Am J Roentgenol 1978; 130: 588-9.

4. Verlaan JJ, Boswijk PF, de Ru JA, et al. Diffuse idiopathic skeletal hyperostosis of the cervical spine: an underestimated cause of dysphagia and airway obstruction. Spine J 2011; 11: 1058-67.

5. Urrutia J, Bono CM. Long-term results of surgical treatment of dysphagia secondary to cervical diffuse idiopathic skeletal hyperostosis. Spine J 2009; 9: e13-7.

6. Warnick C, Sherman MS, Lesser RW. Aspiration pneu- monia due to diffuse cervical hyperostosis. Chest 1990; 98: 763-4.

7. Burkus JK. Esophageal obstruction secondary to diffuse idiopathic skeletal hyperostosis. Orthopedics 1988; 11 : 717-20.

8. Ozgocmen S, Kiris A, Kocakoc E, et al. Osteophyteinduced dysphagia: report of three cases. Joint Bone Spine 2002; 69: 226-9.

9. Stuart D. Dysphagia due to cervical osteophytes. A description of five patients and a review of the literature. Int Orthop 1989; 13: 95-9.

10. Verstraete WL, De Cauwer HG, Verhulst D, et al. Vocal cord immobilisation in diffuse idiopathic skeletal hyperostosis (DISH). Acta Otorhinolaryngol Belg 1998; 52: 79-84.

11. Atzeni F, Sarzi-Puttini P, Bevilacqua M. Calcium deposition and associated chronic diseases (atherosclerosis, diffuse idiopathic skeletal hyperostosis, and others). Rheum Dis Clin North Am 2006; 32: 413-26, viii.

12. Goh PY, Dobson M, Iseli T, et al. Forestier's disease presenting with dysphagia and dysphonia. J Clin Neurosci 2010; 17: 1336-8.

13. Lecerf $\mathrm{P}$, Malard O. How to diagnose and treat symptomatic anterior cervical osteophytes? Eur Ann Otorhinolaryngol Head Neck Dis 2010; 127: 111-6.

14. Humphreys SC, Eck JC, Hodges SD, et al. Preliminary experience with a new surgical treatment for dysphagia due to anterior cervical osteophytes. J Surg Orthop Adv 2004; 13: 106-9.

15. Yee C, Wong HY, Fewer HD, et al. Two cases of dysphagia due to cervical spine osteophytes successfully treated surgically. Can Med Assoc J 1985; 132: 810-2.

16. Sobol SM, Rigual NR. Anterolateral extrapharyngeal approach for cervical osteophyte-induced dysphagia. Literature review. Ann Otol Rhinol Laryngol 1984; 93 : 498-504.

17. Uppal S, Wheatley AH. Transpharyngeal approach for the treatment of dysphagia due to Forestier's disease. J Laryngol Otol 1999; 113: 366-8.

18. Westerveld LA, van Ufford HM, Verlaan JJ, et al. The prevalence of diffuse idiopathic skeletal hyperostosis in an outpatient population in The Netherlands. J Rheumatol 2008; 35: 1635-8. 